\title{
ADDENDUMS TO "GENERALIZED FOURIER-FEYNMAN TRANSFORM AND SEQUENTIAL TRANSFORMS ON FUNCTION SPACE"
}

\author{
Jae Gil Choi and Seung Jun Chang
}

The authors make a correction to the above article, published in Journal of the Korean Mathematical Society 49 (2012), no. 5, pp. 1065-1082, available at dx.doi.org/10.4134/JKMS.2012.49.5.1065.

We presented Theorems 5.3 and 5.4 to establish the existences of the sequential $\mathcal{P}$-transform and the sequential $\mathcal{N}$-transform of the functionals on the function space $C_{a, b}[0, T]$, which is represented by the Fourier transform of a complex measure in the Banach algebra $\mathcal{M}\left(\mathbb{R}^{n}\right)$. It is the core point in the proof of Theorem 5.3 that the dominated convergence theorem in [1, Theorem 4.17 , p. 92] can be used. The rest of the proof of Theorem 5.3 to be applied the dominated convergence theorem will be taken same techniques in the proof of Theorem 4.2 with $T_{\lambda_{l}}(F)(y)$ replaced with $\int_{C_{a, b}[0, T]} F(y+x) W_{\vec{e}}\left(\lambda_{l} ; x\right) d \mu(x)$, definitely.

In the paper, for $\lambda \in \widetilde{\mathbb{C}}_{+}=\{\lambda \in \mathbb{C}: \lambda \neq 0$ and $\operatorname{Re}(\lambda) \geq 0\}, \lambda^{-1 / 2}$ was chosen to be the principal square root of $\lambda$ so that $\left|\operatorname{Arg}\left(\lambda^{1 / 2}\right)\right| \leq \pi / 4$. To apply the dominated convergence theorem, we considered the region

$$
\begin{aligned}
\Gamma_{q_{0}}^{+} \equiv \Gamma_{q_{0}} & =\left\{\lambda \in \widetilde{\mathbb{C}}_{+}:\left|\operatorname{Im}\left(\lambda^{-1 / 2}\right)\right|<\left(2 q_{0}\right)^{-1 / 2}\right\} \\
& =\left\{\lambda \in \widetilde{\mathbb{C}}_{+}: \lambda=\alpha+i \beta, \sqrt{\frac{\sqrt{\alpha^{2}+\beta^{2}}-\alpha}{2\left(\alpha^{2}+\beta^{2}\right)}}<\frac{1}{\sqrt{2 q_{0}}}\right\},
\end{aligned}
$$

where $q_{0}$ is a positive real number. If $q$ is a real number with $|q|>q_{0}$, then by a close examination, we have the following:

- $\Gamma_{q_{0}}^{+}$is an open neighborhood of $-i q$ in $\widetilde{\mathbb{C}}_{+}$(more precisely, $-i q$ is an element of $\partial \Gamma_{q_{0}}^{+}$, the boundary of $\Gamma_{q_{0}}^{+}$in $\left.\widetilde{\mathbb{C}}_{+}\right)$;

- $(-i q)^{-1 / 2}=1 / \sqrt{2|q|}+i \operatorname{sign}(q) / \sqrt{2|q|}$; and

- for $\lambda \in \widetilde{\mathbb{C}}_{+}$with $\lambda=\alpha+i \beta$,

$$
\lambda^{-1 / 2} \equiv\left(\lambda^{-1}\right)^{1 / 2}=\sqrt{\frac{\sqrt{\alpha^{2}+\beta^{2}}+\alpha}{2\left(\alpha^{2}+\beta^{2}\right)}}-i \operatorname{sign}(\beta) \sqrt{\frac{\sqrt{\alpha^{2}+\beta^{2}}-\alpha}{2\left(\alpha^{2}+\beta^{2}\right)}}
$$

Received June 22, 2014.

2010 Mathematics Subject Classification. 28C20, 60J65.

Key words and phrases. sequential $\mathcal{P}$-transform, sequential $\mathcal{N}$-transform. 
and

$$
(-\lambda)^{-1 / 2} \equiv\left(-\lambda^{-1}\right)^{1 / 2}=\sqrt{\frac{\sqrt{\alpha^{2}+\beta^{2}}-\alpha}{2\left(\alpha^{2}+\beta^{2}\right)}}+i \operatorname{sign}(\beta) \sqrt{\frac{\sqrt{\alpha^{2}+\beta^{2}}+\alpha}{2\left(\alpha^{2}+\beta^{2}\right)}},
$$

where $\operatorname{sign}(\beta)=1$ if $\beta \geq 0$ and $\operatorname{sign}(\beta)=-1$ if $\beta<0$.

Thus, given a sequence $\left\{\lambda_{l}\right\}$ in $\mathbb{C}_{+}$which converges to $-i q, \lambda_{l}^{-1 / 2}$ is in $\Gamma_{q_{0}}^{+}$for all but a finite number of values of $l$. In the first line on p. 1074, the inequality ' $\left|\operatorname{Im}\left(\lambda^{-1 / 2}\right)\right|<1 / \sqrt{\left|q_{0}\right|}$ ' should be replaced by ' $\left|\operatorname{Im}\left(\lambda^{-1 / 2}\right)\right|<1 / \sqrt{2\left|q_{0}\right|}$ '. Thus the proof of the existence of the sequential $\mathcal{P}$-transform has no problem.

However, we have found the fact that the region $\Gamma_{q_{0}}^{+}$in $\widetilde{\mathbb{C}}_{+}$cannot be used for the proof of the existence of the sequential $\mathcal{N}$-transform in Theorem 5.4. When we use the region $\Gamma_{q_{0}}^{+}$to apply the dominated convergence theorem in the proof Theorem 5.4, we shall be faced with an illogical situation. Thus we suggest that for the proof of Theorem 5.4, one can use the neighborhood $\Gamma_{q_{0}}^{-}$ of $-i q$ given by

$$
\begin{aligned}
\Gamma_{q_{0}}^{-} & =\left\{\lambda \in \widetilde{\mathbb{C}}_{+}:\left|\operatorname{Im}\left((-\lambda)^{-1 / 2}\right)\right|<\left(2 q_{0}\right)^{-1 / 2}\right\} \\
& =\left\{\lambda \in \widetilde{\mathbb{C}}_{+}: \lambda=\alpha+i \beta, \sqrt{\frac{\sqrt{\alpha^{2}+\beta^{2}}+\alpha}{2\left(\alpha^{2}+\beta^{2}\right)}}<\frac{1}{\sqrt{2 q_{0}}}\right\} .
\end{aligned}
$$

The region $\Gamma_{q_{0}}^{-}$is also an open neighborhood of $-i q$ in $\widetilde{\mathbb{C}}_{+}$. Also $-i q$ is an element of $\partial \Gamma_{q_{0}}^{-}$. By the definitions of the regions $\Gamma_{q_{0}}^{+}$and $\Gamma_{q_{0}}^{-}$, one can see that $\Gamma_{q_{0}}^{-} \subsetneq \Gamma_{q_{0}}^{+}$.

Also the open neighborhood $\Gamma_{q_{0}}^{-}$of $-i q$ in $\widetilde{\mathbb{C}}_{+}$can be used in the proofs of Theorems 4.2 and 5.3 because

$$
\left|\operatorname{Im}\left(\lambda^{-1 / 2}\right)\right|<\left|\operatorname{Im}\left((-\lambda)^{-1 / 2}\right)\right|
$$

for all $\lambda \in \mathbb{C}_{+}=\{\lambda \in \mathbb{C}: \operatorname{Re}(\lambda)>0\}$.

\title{
References
}

[1] H. L. Royden, Real Analysis, Third edition, Macmillan, 1988.

\author{
JAE GIL ChOI \\ Department of Mathematics \\ DANKOOK UNIVERSITY \\ Cheonan 330-714, Korea \\ E-mail address: jgchoi@dankook.ac.kr \\ Seung Jun Chang \\ Department of Mathematics \\ DANKOOK UNIVERSITY \\ Cheonan 330-714, Korea \\ E-mail address: sejchang@dankook.ac.kr
}

